\title{
All-epiphyseal Anterior Cruciate Ligament Reconstruction in Skeletally Immature Patients
}

\author{
J. Todd R. Lawrence MD, PhD, Andrea L. Bowers MD, \\ Jonathan Belding MD, Stephanie R. Cody BS, \\ Theodore J. Ganley MD
}

Received: 18 May 2009/Accepted: 25 January 2010/Published online: 20 February 2010

(C) The Association of Bone and Joint Surgeons $\mathbb{R} 2010$

\begin{abstract}
Background Treating ACL injuries in prepubescent patients requires balancing the risk of chondral and meniscal injuries associated with delaying treatment against the risk of growth disturbance from early surgical reconstruction. Multiple physeal respecting techniques have been described to address this vulnerable population; however, none restore the native ACL attachments while keeping the graft and fixation entirely in the epiphysis.

Description of Technique We describe a technique of allepiphyseal ACL reconstruction for use in prepubescent skeletally immature patients. Intraoperative CT scanning with three-dimensional (3-D) reconstruction was used to confirm the precise localization of the all-epiphyseal femoral and tibial tunnels. The femoral tunnel is drilled
\end{abstract}

Each author certifies that he or she has no commercial associations (eg, consultancies, stock ownership, equity interest, patent/licensing arrangements, etc) that might pose a conflict of interest in connection with the submitted article.

Each author certifies that his or her institution approved the human protocol for this investigation, that all investigations were conducted in conformity with ethical principles of research, and that informed consent for participation in the study was obtained.

This work was performed at The Children's Hospital of Philadelphia.

J. T. R. Lawrence, S. R. Cody, T. J. Ganley ( $\bigotimes)$

Division of Orthopaedic Surgery, Children's Hospital of Philadelphia, Wood Building, 2nd Floor, 34th Street and Civic Center Boulevard, Philadelphia, PA 19104-4399, USA

e-mail: ganley@email.chop.edu

A. L. Bowers, T. J. Ganley

Department of Orthopaedic Surgery, University of Pennsylvania, Philadelphia, PA, USA

J. Belding

Case Western Reserve School of Medicine, Cleveland, OH, USA entirely in the epiphysis of the lateral femoral condyle. The tibial tunnel is drilled from inside-out to the level of the tibial physis using a retrograde drill. Fixation of the soft tissue graft is achieved with a retrograde interference screw in the tibia and an interference screw in the femur.

Patients and Methods Case examples are presented for three boys aged 10-12, Tanner Stage 1 development, with a minimum followup of 1 year.

Results All three patients had stable knees based on Lachman and KT-1000 testing and no evidence of growth disturbance. All had full ROM and symmetric strength for knee flexion and extension. All patients returned to their sports activities using a custom ACL brace.

Conclusions Although longer-term followup will be necessary, this technique provides for an anatomic allepiphyseal-based ACL reconstruction using intraoperative 3-D imaging to minimize the risk of growth disturbance.

Level of Evidence Level IV, therapeutic study. See the Guidelines for Authors for a complete description of levels of evidence.

\section{Introduction}

ACL injuries are being seen with increasing frequency in skeletally immature patients $[22,24]$. The natural history of nonoperative treatment of this injury is poor with chronic instability leading to chondral and meniscal injuries $[1,16$, $20,28,31,32,35]$. However, traditional surgical stabilization, with grafts placed through tunnels drilled across the physis, risks iatrogenic damage to the physis, which may produce a growth disturbance. This risk increases with increasing amounts of growth remaining.

Growth disturbances after ACL reconstruction in this population have been associated with some form of graft 
fixation or bony graft material across the physis or involve damage to the perichondral ring [24-26]. Based on animal models $[12,36,39]$ and these clinical examples, the following general principles now are accepted [23, 24]: fixation should not cross the physis, drill holes across the physis should be centrally located and as small as possible, and tensioning a soft tissue graft across a physis should be avoided if a large amount of growth remains.

Multiple techniques for surgical stabilization of the ACL-deficient knee in prepubescent patients have been described [1-11, 13-16, 18, 19, 21, 27-30, 34, 37, 38, 40, $42,43]$. All try to balance the advantages of an anatomic reconstruction with the risk of physeal injury and have advantages and disadvantages. Although techniques have been described for reconstructing the ACL without drilling tunnels across the physis, none of these techniques provide for a reconstruction that places the graft in the native footprint of the ACL while strictly adhering to the principles described by using all-epiphyseal fixation of the graft.

We describe a new technique for ACL reconstruction in prepubescent skeletally immature patients that adheres to the generally accepted principles of adult ACL reconstruction, but theoretically minimizes the risk to the growing physis by avoiding any fixation, drill holes, or tensioning of the graft across the physis.

\section{Surgical Technique}

Arthroscopy is performed through standard anteromedial working and anterolateral viewing arthroscopy portals. The diagnosis of complete intrasubstance tear of the ACL is confirmed. All associated meniscus disorders are addressed. We routinely use a combination of all-inside, inside-out, and outside-in techniques as dictated by the tear.

The residual ACL is removed and the tibial and femoral footprints are identified and cleared of excess soft tissue. An outside-in femoral guide, set at $95^{\circ}$, is placed through the medial portal into the notch. A $1.5-$ to $2-\mathrm{cm}$ incision is made over the lateral femur just anterior and distal to the lateral epicondyle and blunt dissection is performed to the level of the bone. The $95^{\circ}$-femoral guide then is used to pass a guide wire parallel to the physis in the epiphysis to the center of the femoral ACL footprint (Fig. 1). The femoral guide is removed, leaving the wire in place.

A Retrodrill (Arthrex, Naples, FL) then is used to drill the all-epiphyseal tibial tunnel (Fig. 1A). Based on the size of the graft, the appropriate size Retrodrill cutting blade is placed over the center of the anatomic footprint of the ACL. The cannulated guide pin for the Retrodrill is advanced to capture the cutting blade from the targeting guide. The Retrodrill is drawn back slowly for a distance of approximately $17 \mathrm{~mm}$, or approximately $3 \mathrm{~mm}$ less than the distance measured on MRI (approximately $20 \mathrm{~mm}$ ) from the tibial ACL footprint to the physis. The position of the Retrodrill and the femoral guide pin are adjusted, if necessary, using fluoroscopic guidance. The scout images from the localization process of the $\mathrm{O}$-arm can be used for this.

A limited-cut, low-dose radiation, intraoperative CT scan then is performed with an O-arm (Medtronic, Inc, Minneapolis, MN) to confirm (1) an all-epiphyseal placement of the tibial drill hole and the femoral guide wire; (2) anatomic entry points for the tunnels; and (3) an adequate distance from the physis to prevent damage (Fig. 2). Once confirmed, the femoral tunnel is drilled using standard cannulated reamers from an outside-to-inside approach. Direct arthroscopic observation of the femoral tunnel then is performed to ensure that the femoral physis has not been violated. The Retrodrill in the tibia is carefully advanced back into the joint. The cutting blade is uncoupled and the cannulated Retrodrill guide pin is left in place.

For graft harvest and preparation, an all soft tissue graft is used. Autologous quadrupled hamstrings, gracilis, and semitendinosus, are preferred; however, according to the preference of the patient and family, allograft tendon can be used. Hamstring tendons are harvested using standard techniques. The graft is prepared by whipstitching the ends of the tendon and then looping them over a Number 5 Ethibond suture (Ethicon, Somerville, NJ). A 20-mm length centered about the looped end also is whipstitched to help prevent graft rolling and damage from the interference screw. The prepared graft is sized, wrapped in damp gauze, and pretensioned to 15 pounds.

For graft passage, the inner portion of the cannulated Retrodrill guide pin is removed and a Fiberstick (Arthrex, Naples, FL) suture passed up the cannulated guide pin to the joint. The suture is retrieved from the femoral tunnel and the cannulated Retrodrill guide pin removed from the tibia (Fig. 1B). The suture limbs from the looped portion of the graft and a Nitinol wire (Arthrex, Naples, FL) for the Retroscrew screwdriver then are shuttled from the femoral side to the tibial side using the previously passed Fiberstick suture (Fig. 1C). The Nitinol wire is retained in the tibial tunnel, retrieved from the femoral tunnel, and brought out the anteromedial portal to place it in the anterior portion of the tibial tunnel. The graft then is advanced into the knee through the femoral tunnel and firmly seated in the tibial tunnel by pulling the Number 5 Ethibond suture on the looped end of the graft (Fig. 1D).

For graft fixation, the Retroscrew screwdriver is advanced up to the tibial tunnel over the Nitinol wire to the joint and positioned anterior to the graft. The Nitinol wire is removed and a Fiberstick suture placed up the screwdriver to the joint and retrieved out the anteromedial portal. An appropriately sized Retroscrew (Arthrex) then is placed on the Fiberstick suture and a mulberry knot tied behind it. For most 
A

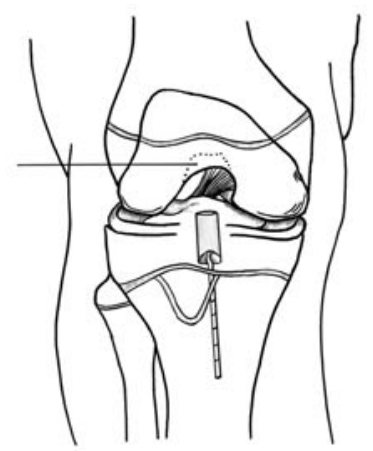

B

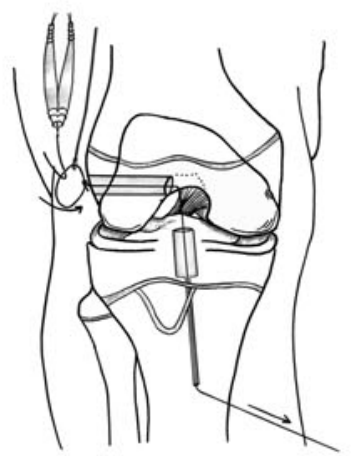

C

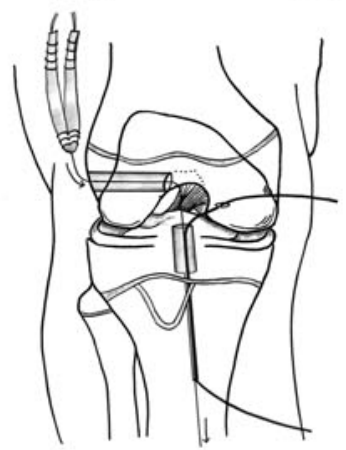

D

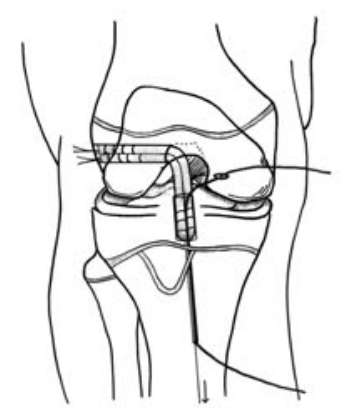

$\mathbf{E}$

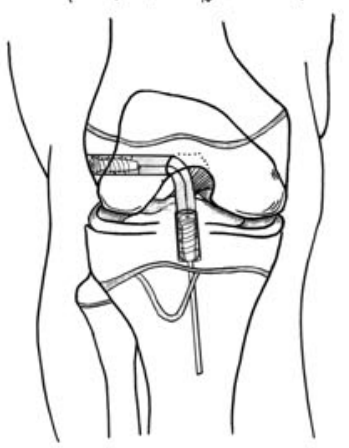

4Fig. 1A-E The all-epiphyseal ACL reconstruction technique is shown. (A) A guide wire is placed in the distal femoral epiphysis parallel to the physis to the center of the femoral attachment point of the ACL. A Retrograde drill (Arthrex, Naples FL) is used in the tibia to create a tunnel in the tibial epiphysis. Proper placement can be confirmed with an intraoperative CT scan. (B) The femoral tunnel is drilled. A passing suture is placed up the cannulated guide pin of the Retrodrill and retrieved out the femoral tunnel. (C) The passing suture is used to bring a Nitinol wire and the lead sutures of the graft through the knee. The femoral end of the Nitinol wire then is retrieved out the medial portal. (D) The lead sutures on the graft are used to pull the graft through the femoral tunnel and into the tibial tunnel. (E) The Nitinol wire then is used to aid in placement of the tibial Retroscrew (Arthrex, Naples FL). The knee is brought into extension and the graft tensioned and secured in the femur with an interference screw.

procedures, a $20 \mathrm{~mm} \times 9-\mathrm{mm}$ Retroscrew has been used in a 9-mm tibial tunnel. Through an expandable cannula, the Retroscrew is advanced into the joint, flipped onto the screwdriver, and screwed into place while holding the graft tensioned and firmly seated in the tibial tunnel. Finally, the knee is cycled numerous times, brought into full extension, and reduced with a gentle posterior drawer. The femoral side is fixed with an interference screw while applying 15 pounds of tension to the graft with a graft tensioner (Fig. 1E). The wounds are irrigated and closed in layers followed by soft dressings and a knee brace locked in maximum extension.

Cryotherapy and continuous passive motion are used for the first 2 weeks postoperatively. Weightbearing is restricted to touch-down weightbearing for 6 weeks with the brace locked in extension for ambulation during that time. Formal physical therapy with an accelerated ACL protocol is initiated approximately 2 weeks postoperatively and continued until approximately 4 months. Modifications are made for the restricted weightbearing status for the first 6 weeks. Given the young age of these patients, it is important to identify therapists who are capable and motivated to work with patients this age. The therapy also is modified according to the patients' maturity and ability to comply with restrictions. Return to sports is allowed approximately 6 to 9 months after surgery when formal strength and functional testing are at least $90 \%$ of the contralateral side. Functional ACL bracing during sports activities is recommended for approximately 2 years after reconstruction. Routine followup with radiographs is performed on a yearly basis until skeletal maturity. Angular deformity is assessed radiographically and clinically and leg length is assessed clinically.

\section{Case Reports}

Three case examples are presented. All of the patients and their families consented to presentation of their cases performed by the senior author (Theodore Ganley, MD). 
Fig. 2A-C (A) This intraoperative photograph shows the knee with the femoral guide-wire and tibial Retrodrill in place in preparation for 3-D intraoperative scanning. 3-D ghost images are obtained. When rotated in real time intraoperatively, accurate and safe localization can be assessed easily, as seen in these (B) lateral and (C) AP views, in relation to the undulating physes. Adjustments can be made at this time based on this imaging and limited rescanning performed to confirm accurate placement.

Patient 1 was a healthy 12-year-old boy who presented to us with a left knee injury that he sustained by a twisting mechanism playing basketball 2 years before. He was diagnosed with a complete rupture of the ACL and was managed by an outside surgeon with a regime of activity modification, bracing, and functional rehabilitation. He continued to experience episodes of instability and therefore was referred to us for additional evaluation and treatment. Physical examination and repeat MRI confirmed the diagnosis of an ACL tear with an associated medial meniscus tear. Understanding the risks and benefits, the patient and his family elected he undergo surgical reconstruction. At the time of reconstruction, Tanner staging revealed Stage 1 physical development and wrist radiographs revealed a bone age of 12 years [17, 41]. An all-epiphyseal reconstruction was performed as detailed previously with allograft posterior tibialis tendon per the preference of the patient and his family. Arthroscopic allinside medial meniscus repair also was performed.

Patient 2 was a healthy 10 -year-old boy who sustained an intrasubstance ACL tear from a minimal contact injury while playing football. This was confirmed with physical examination and MRI. He experienced recurrent instability with daily activity despite bracing. Understanding the risks and benefits, the patient and his family elected he undergo surgical reconstruction using the all-epiphyseal technique with autograft hamstrings as described previously. His bone age based on wrist radiographs was 10 years and Tanner staging revealed Stage 1 physical development at the time of reconstruction.

Patient 3 was a healthy 12-year-old boy who sustained an injury to his left knee during football practice. An intrasubstance ACL tear and a lateral meniscus tear were confirmed with physical examination and MRI. He also experienced recurrent instability with daily activity despite bracing, and understanding the risks and benefits, he and his parents elected he undergo surgical reconstruction. At the time of reconstruction, wrist radiographs showed a bone age of 12 years and he had Tanner Stage 1 physical development. He underwent ACL reconstruction using the all-epiphyseal technique with autograft hamstrings as described previously and an all-inside lateral meniscus repair.

At 1-year followup, no patient had any evidence of an angular deformity measured radiographically or any
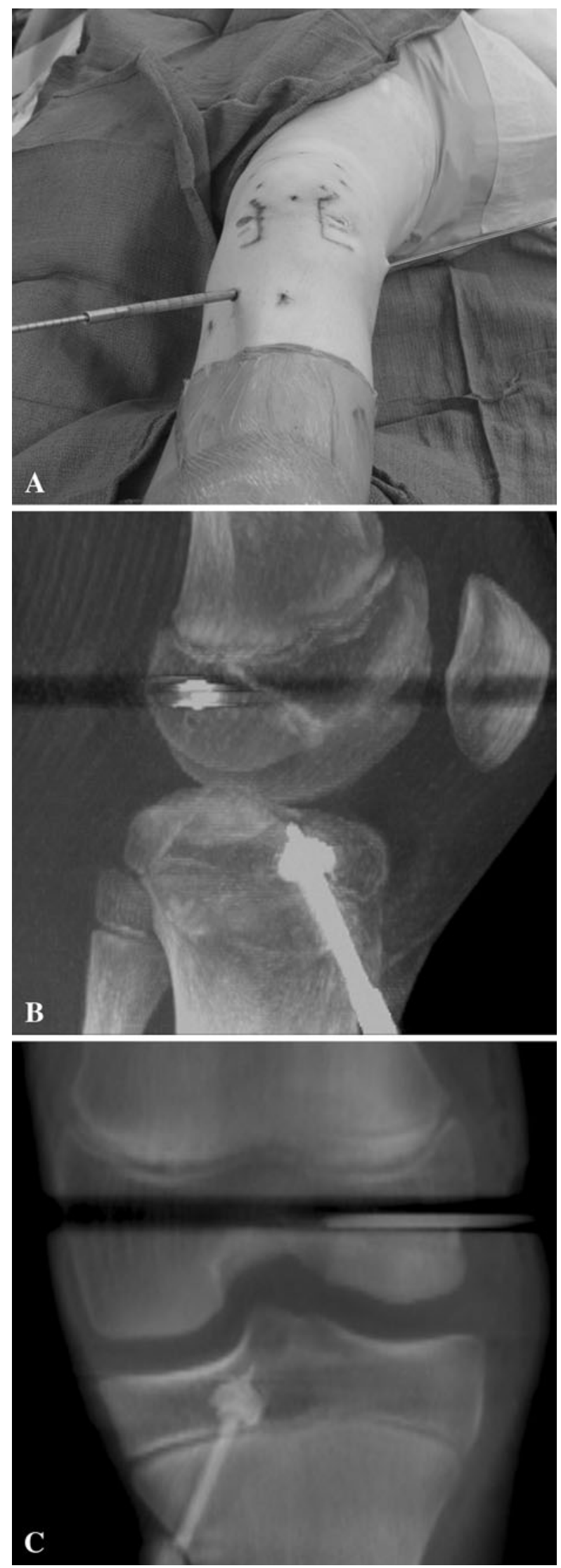
Fig. 3A-B These (A) AP and (B) lateral radiographs were obtained 6 months postoperatively.
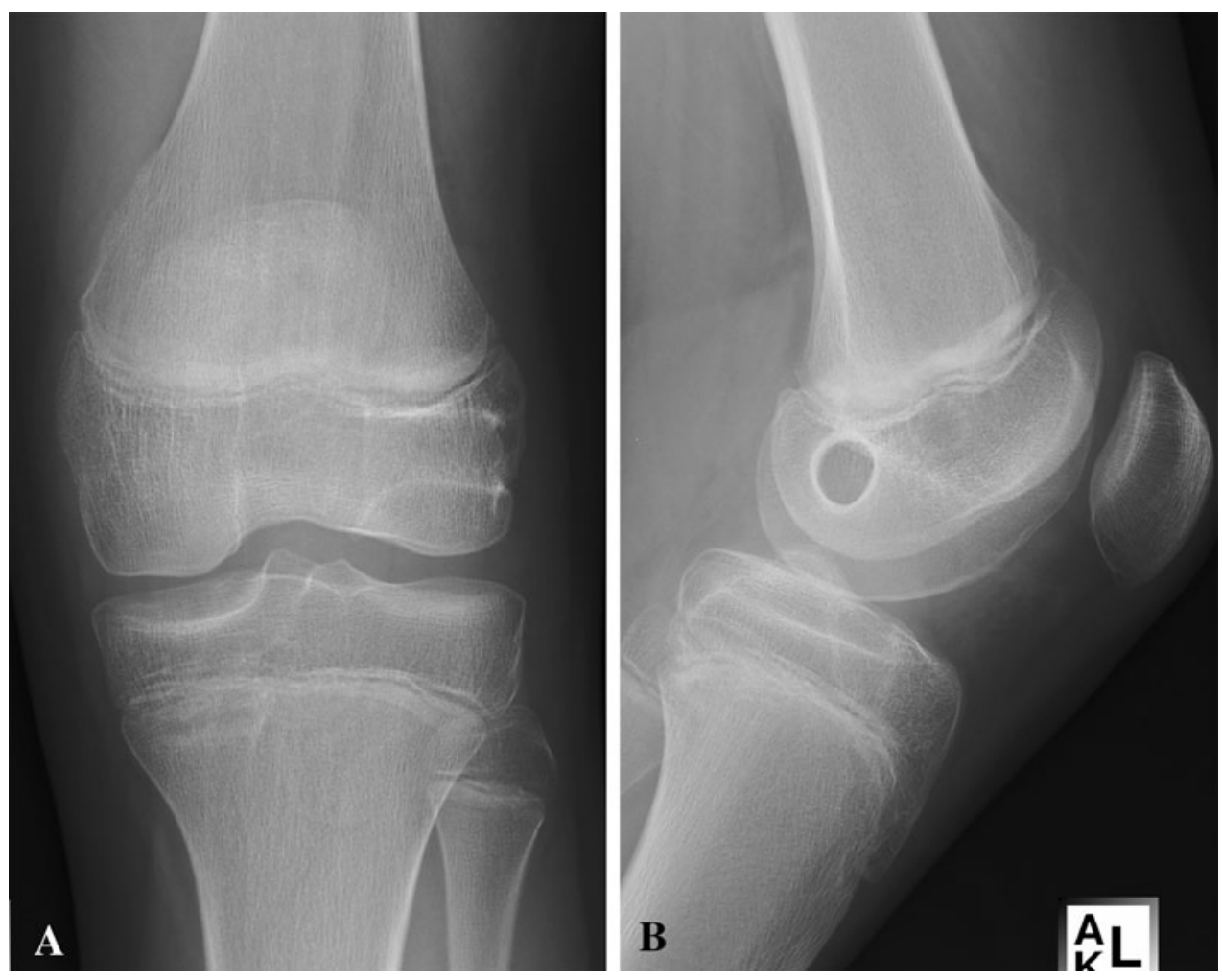

discrepancy in the leg length measured clinically (Fig. 3). All had full ROM and symmetric strength for knee flexion and extension. Lachman and pivot shift testing were normal, and KT-1000 arthrometry showed no more than $1 \mathrm{~mm}$ side-to-side difference with manual maximum displacement testing. Strength testing and functional dynamic testing (ie, hop test) were greater than $90 \%$ compared with the uninjured side. All patients returned to their sports activities using a custom ACL brace. No evidence of meniscal retear or repair failure was observed.

\section{Discussion}

Multiple physeal-sparing techniques for ACL reconstruction have been described. The technique described by Guzzanti et al. involves an eccentric 6-mm tibial tunnel drilled from the central proximal tibia, proximal to the physis, through the epiphysis and to the tibial eminence [18]. The femoral attachment is prepared with a shallow drill site in the distal epiphysis. An autograft hamstring graft is left attached distally, brought into the knee through the tibial tunnel, looped over and fixed to the femur over a staple. The graft is brought out of the tibial tunnel and sewn to itself and to the anterior tibial periosteum distal to the physis. This procedure was tolerated well by five skeletally immature patients with no reported cases of growth disturbance and no issues of recurrent instability at a minimum followup of 4 years (average, 5.7 years; range, 4-7 years).

Anderson described a similar technique using intraoperative fluoroscopic imaging to ensure all-epiphyseal placement of distal femoral and proximal tibial tunnels $[2,3]$. The femoral tunnel is drilled using fluoroscopic guidance in a similar fashion to the tunnel used in the technique described here, being all epiphyseal and parallel to the distal femoral physis. The tibial tunnel is similar to that used by Guzzanti et al. [18]. The doubled hamstring autograft is looped over an Endobutton and washer on the femoral side and, similar to the Guzzanti technique, secured distal to the proximal tibial physis with a screw post $[2,3,18]$. Surgeries were performed on 12 patients with a mean followup of 4.1 years. The authors reported good objective clinical results with no failures.

Micheli et al. [30] and Kocher et al. [24] reported on a physeal-sparing technique in which a strip of iliotibial band is harvested, left attached at Gerdy's tubercle, passed through the notch of the knee in the over-the-top position, and then out of the anterior aspect of the knee under the transverse meniscal ligament through a shallow trough in the anterior tibial plateau. The graft is secured to the anterior tibial periosteum distal to the physis. Although this is a nonanatomic reconstruction, good functional outcomes have been reported with a low failure rate approaching those of adult reconstructions. 
The surgical technique we describe uses new technology for localizing tunnels, drilling tunnels, and graft fixation to achieve an all-epiphyseal ACL reconstruction. Although this is a procedure recommended for experienced arthroscopists, we believe these incremental advances have made the procedure less demanding technically. In addition, we believe it offers several advantages over the currently described techniques.

The femoral tunnel described by Anderson [2, 3] is similar to the tunnel described here; however, we favor the placement and fixation methods we describe. The undulating nature of the distal femoral physis makes it difficult to assess the true relationship of the guide wire to the physis with fluoroscopic imaging only. Intraoperative CT with 3-D reconstruction does not necessarily help with placement of the guide wire. However, it does allow for accurate assessment of the distance to the physis along the entire length of the guide wire. Thus, although intraoperative 3-D imaging is not necessary, we favor the more accurate confirmation of placement afforded by the 3-D imaging. In terms of fixation on the femoral side, we use a 23-mm interference screw with the graft under tension. Tunnel length in the femur has not been an issue. Fixation with a staple, as in the technique of Guzzanti et al. [18], seems less biomechanically sound, and in our experience, is less amenable to placement in the proper anatomic location. Although fixation with an Endobutton over a washer on the lateral cortex, as in the Anderson technique $[2,3]$, is as biomechanically sound as the interference screw fixation described here, it provides a longer graft length for potential stretch of the all soft tissue graft [2,3].

In terms of the tibial tunnel, although the techniques of Guzzanti et al. [18] and Anderson [2, 3] are more anatomic than the techniques of Micheli et al. [30] and Kocher et al. $[23,24]$, they are more technically demanding and have a lower margin of error $[2,3,18,22,23,30]$. The obliquity of the tibial tunnel makes the intraarticular opening oblong and can accentuate any misplacement of the tunnel. We believe the retrodrilling technique described here allows for accurate placement of a tibial tunnel that is more vertically and perhaps more centrally located [33]. The central portion of the tibial physis is crossed with the $3-\mathrm{mm}$ guide wire of the Retrodrill, but because no fixation crosses the physis and the insult to the physis is central and small, the risk of inducing a growth disturbance is minimized. However, the need for the tibial tunnel to be at least $20 \mathrm{~mm}$ to accommodate the tibial Retroscrew does limit the size of the knees that can be addressed with this technique.

The main disadvantage of this technique is that it requires advanced technology and the use of the O-arm in the operative suite. Operative time for this procedure is only slightly longer than that of a conventional reconstruction. It takes no longer to prepare the knee, drill the tunnels, or pass and fix the graft than a conventional ACL reconstruction. With experience and a well-trained staff, intraoperative CT imaging can be obtained in 10 to 15 minutes and provides 3-D imaging for the tibial and femoral tunnels. In our experience, fluoroscopic imaging with perfect alignment in two planes to properly observe the coronal and sagittal undulations of the distal femoral physis in relation to the guide wire takes approximately the same amount of time. Therefore, there are no increased direct costs to the patient or in terms of OR time. There is certainly an upfront cost to the institution for this instrumentation, but this can be split over the many services that use the scanner. However, once available, there are not increased costs for its preferential use over conventional fluoroscopy. Therefore, it is reasonable for those without access to intraoperative 3-D imaging to perform this technique using fluoroscopic guidance. Thus, if available, given the increased confidence in the safety of the tunnel placement, we believe use of the $\mathrm{O}$-arm is justified.

Unfortunately, for our young athletes, the incidence of primary ACL injuries in prepubescent skeletally immature patients is increasing $[22,24]$. The poor natural history of intermediate and long-term nonoperative treatment of this injury $[1,16,20,28,31,32,35]$ has resulted in physealrespecting ACL reconstruction being a more common operative procedure. In this population, however, every effort must be made to minimize growth arrest. Current instrumentation and technology has allowed us to improve on existing techniques by creating an all-epiphyseal ACL reconstruction using a more traditional tibial tunnel with confirmation of graft and tunnel placement using 3-D imaging. We believe this novel procedure offers the arthroscopist familiar with the techniques described the opportunity to manage the ACL-deficient but skeletally immature patient with a physeal-respecting reconstruction.

Acknowledgments We thank Nick Wright for support in the development of this technique.

\section{References}

1. Aichroth PM, Patel DV, Zorrilla P. The natural history and treatment of rupture of the anterior cruciate ligament in children and adolescents: a prospective review. J Bone Joint Surg Br. 2002;84:38-41.

2. Anderson AF. Transepiphyseal replacement of the anterior cruciate ligament in skeletally immature patients: a preliminary report. J Bone Joint Surg Am. 2003;85:1255-1263.

3. Anderson AF. Transepiphyseal replacement of the anterior cruciate ligament using quadruple hamstring grafts in skeletally immature patients. J Bone Joint Surg Am. 2004;86(suppl 1 pt 2): 201-209.

4. Andrews M, Noyes FR, Barber-Westin SD. Anterior cruciate ligament allograft reconstruction in the skeletally immature athlete. Am J Sports Med. 1994;22:48-54. 
5. Angel KR, Hall DJ. Anterior cruciate ligament injury in children and adolescents. Arthroscopy. 1989;5:197-200.

6. Aronowitz ER, Ganley TJ, Goode JR, Gregg JR, Meyer JS. Anterior cruciate ligament reconstruction in adolescents with open physes. Am J Sports Med. 2000;28:168-175.

7. Bisson LJ, Wickiewicz T, Levinson M, Warren R. ACL reconstruction in children with open physes. Orthopedics. 1998;21: 659-663.

8. Brief LP. Anterior cruciate ligament reconstruction without drill holes. Arthroscopy. 1991;7:350-357.

9. Clanton TO, DeLee JC, Sanders B, Neidre A. Knee ligament injuries in children. J Bone Joint Surg Am. 1979;61:1195-1201.

10. DeLee JC, Curtis R. Anterior cruciate ligament insufficiency in children. Clin Orthop Relat Res. 1983;172:112-118.

11. Edwards PH, Grana WA. Anterior cruciate ligament reconstruction in the immature athlete: long-term results of intra-articular reconstruction. Am J Knee Surg. 2001;14:232-237.

12. Edwards TB, Greene CC, Baratta RV, Zieske A, Willis RB. The effect of placing a tensioned graft across open growth plates: a gross and histologic analysis. J Bone Joint Surg Am. 2001;83: 725-734.

13. Engebretsen L, Benum P, Sundalsvoll S. Primary suture of the anterior cruciate ligament: a 6-year follow-up of 74 cases. Acta Orthop Scand. 1989;60:561-564.

14. Engebretsen L, Svenningsen S, Benum P. Poor results of anterior cruciate ligament repair in adolescence. Acta Orthop Scand. 1988;59:684-686.

15. Fuchs R, Wheatley W, Uribe JW, Hechtman KS, Zvijac JE, Schurhoff MR. Intra-articular anterior cruciate ligament reconstruction using patellar tendon allograft in the skeletally immature patient. Arthroscopy. 2002;18:824-828.

16. Graf BK, Lange RH, Fujisaki CK, Landry GL, Saluja RK. Anterior cruciate ligament tears in skeletally immature patients: meniscal pathology at presentation and after attempted conservative treatment. Arthroscopy. 1992;8:229-233.

17. Greulich WW, Pyle SI. Radiographic Atlas of Skeletal Development of the Hand and Wrist. Ed 2. Stanford, CA: Stanford University Press; 1959.

18. Guzzanti V, Falciglia F, Stanitski CL. Physeal-sparing intraarticular anterior cruciate ligament reconstruction in preadolescents. Am J Sports Med. 2003;31:949-953.

19. Guzzanti V, Falciglia F, Stanitski CL. Preoperative evaluation and anterior cruciate ligament reconstruction technique for skeletally immature patients in Tanner stages 2 and 3. Am J Sports Med. 2003;31:941-948.

20. Janarv PM, Nystrom A, Werner S, Hirsch G. Anterior cruciate ligament injuries in skeletally immature patients. J Pediatr Orthop. 1996;16:673-677.

21. Kim SH, Ha KI, Ahn JH, Chang DK. Anterior cruciate ligament reconstruction in the young patient without violation of the epiphyseal plate. Arthroscopy. 1999;15:792-795.

22. Kocher MS, Garg S, Micheli LJ. Physeal sparing reconstruction of the anterior cruciate ligament in skeletally immature prepubescent children and adolescents. J Bone Joint Surg Am. 2005;87:2371-2379.

23. Kocher MS, Hovis WD, Curtin MJ, Hawkins RJ. Anterior cruciate ligament reconstruction in skeletally immature knees: an anatomical study. Am J Orthop. 2005;34:285-290.

24. Kocher MS, Saxon HS, Hovis WD, Hawkins RJ. Management and complications of anterior cruciate ligament injuries in skeletally immature patients: survey of the Herodicus Society and The ACL Study Group. J Pediatr Orthop. 2002;22:452-457.
25. Koman JD, Sanders JO. Valgus deformity after reconstruction of the anterior cruciate ligament in a skeletally immature patient: a case report. J Bone Joint Surg Am. 1999;81:711-715.

26. Lipscomb AB, Anderson AF. Tears of the anterior cruciate ligament in adolescents. J Bone Joint Surg Am. 1986;68:19-28.

27. Matava MJ, Siegel MG. Arthroscopic reconstruction of the ACL with semitendinosus-gracilis autograft in skeletally immature adolescent patients. Am J Knee Surg. 1997;10:60-69.

28. McCarroll JR, Rettig AC, Shelbourne KD. Anterior cruciate ligament injuries in the young athlete with open physes. Am J Sports Med. 1988;16:44-47.

29. McCarroll JR, Shelbourne KD, Porter DA, Rettig AC, Murray S. Patellar tendon graft reconstruction for midsubstance anterior cruciate ligament rupture in junior high school athletes: an algorithm for management. Am J Sports Med. 1994;22:478-484.

30. Micheli LJ, Rask B, Gerberg L. Anterior cruciate ligament reconstruction in patients who are prepubescent. Clin Orthop Relat Res. 1999;364:40-47.

31. Millett PJ, Willis AA, Warren RF. Associated injuries in pediatric and adolescent anterior cruciate ligament tears: does a delay in treatment increase the risk of meniscal tear? Arthroscopy. 2002;18:955-959.

32. Mizuta H, Kubota K, Shiraishi M, Otsuka Y, Nagamoto N, Takagi K. The conservative treatment of complete tears of the anterior cruciate ligament in skeletally immature patients. $J$ Bone Joint Surg Br. 1995;77:890-894.

33. Morgan CD, Stein DA, Leitman EH, Kalman VR. Anatomic tibial graft fixation using a retrograde bio-interference screw for endoscopic anterior cruciate ligament reconstruction. Arthroscopy. 2002;18:E38.

34. Parker AW, Drez D Jr, Cooper JL. Anterior cruciate ligament injuries in patients with open physes. Am J Sports Med. 1994;22:44-47.

35. Pressman AE, Letts RM, Jarvis JG. Anterior cruciate ligament tears in children: an analysis of operative versus nonoperative treatment. J Pediatr Orthop. 1997;17:505-511.

36. Seil R, Pape D, Kohn D. The risk of growth changes during transphyseal drilling in sheep with open physes. Arthroscopy. 2008;24:824-833.

37. Shelbourne KD, Gray T, Wiley BV. Results of transphyseal anterior cruciate ligament reconstruction using patellar tendon autograft in tanner stage 3 or 4 adolescents with clearly open growth plates. Am J Sports Med. 2004;32:1218-1222.

38. Simonian PT, Metcalf MH, Larson RV. Anterior cruciate ligament injuries in the skeletally immature patient. Am J Orthop. 1999;28:624-628.

39. Stadelmaier DM, Arnoczky SP, Dodds J, Ross H. The effect of drilling and soft tissue grafting across open growth plates: a histologic study. Am J Sports Med. 1995;23:431-435.

40. Stanitski CL. Anterior cruciate ligament injury in the skeletally immature patient: diagnosis and treatment. J Am Acad Orthop Surg. 1995;3:146-158.

41. Tanner JM, Whitehouse RH. Clinical longitudinal standards for height, weight, height velocity, weight velocity, and stages of puberty. Arch Dis Child. 1976;51:170-179.

42. Vahasarja V, Kinnuen P, Serlo W. Arthroscopy of the acute traumatic knee in children: prospective study of 138 cases. Acta Orthop Scand. 1993;64:580-582.

43. Volpi P, Galli M, Bait C, Pozzoni R. Surgical treatment of anterior cruciate ligament injuries in adolescents using doublelooped semitendinosus and gracilis tendons: supraepiphysary femoral and tibial fixation. Arthroscopy. 2004;20:447-449. 